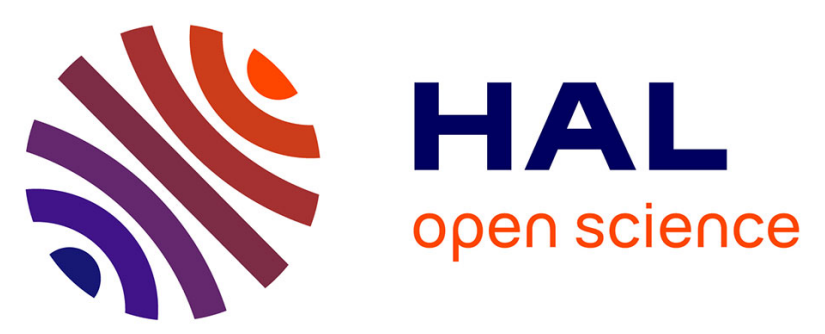

\title{
Experimental and theoretical studies of intergranular strain in an alpha titanium alloy during plastic deformation
}

David Gloaguen, Guy Oum, Vincent Legrand, Jamal Fajoui, Samuel Branchu

\section{- To cite this version:}

David Gloaguen, Guy Oum, Vincent Legrand, Jamal Fajoui, Samuel Branchu. Experimental and theoretical studies of intergranular strain in an alpha titanium alloy during plastic deformation. Acta Materialia, 2013, 61 (15), pp.5779-5790. 10.1016/j.actamat.2013.06.022 . hal-01006837

\section{HAL Id: hal-01006837 \\ https://hal.science/hal-01006837}

Submitted on 3 Mar 2017

HAL is a multi-disciplinary open access archive for the deposit and dissemination of scientific research documents, whether they are published or not. The documents may come from teaching and research institutions in France or abroad, or from public or private research centers.
L'archive ouverte pluridisciplinaire HAL, est destinée au dépôt et à la diffusion de documents scientifiques de niveau recherche, publiés ou non, émanant des établissements d'enseignement et de recherche français ou étrangers, des laboratoires publics ou privés.

\section{(c)(1)}

Distributed under a Creative Commons Attribution| 4.0 International License 


\title{
Experimental and theoretical studies of intergranular strain in an alpha titanium alloy during plastic deformation
}

\author{
D. Gloaguen, G. Oum, V. Legrand, J. Fajoui, S. Branchu \\ Institut de Recherche en Génie Civil et Mécanique (UMR CNRS 6183), LUNAM Université, Université de Nantes, Centrale Nantes, CRTT, 37 Boulevard \\ de l'Université, BP 406, 44602 Saint-Nazaire Cedex, France
}

Two complementary methods were used to analyse the deformation mechanisms involved in the plasticity of commercially pure titanium during compressive loading: neutron diffraction and the self-consistent model. The strain response of 15 crystallographic planes was tracked during the measurement, in directions both parallel and transverse to the straining direction, providing new insight into the mechanical behaviour of the polycrystal. The neutron diffraction results show evidence of tensile twins in the titanium alloy studied. The twin volume fraction was determined from the change in crystallographic texture. The influence and the role of plastic anisotropy were also studied and explained in this work. Good agreement was found between the experimental and predicted results.

Keywords: Titanium; Plasticity; Neutron diffraction; EPSC model

\section{Introduction}

Titanium has a low density, high resistance to corrosion and a high strength-to-density ratio as well as other attractive mechanical properties. These properties have increased its use in chemical plant construction as well as aerospace technology. Titanium alloys ( $\alpha$-phase) have a hexagonal close packed (hcp) structure at room temperature. Like most hcp metals, they are anisotropic in their properties at mesoscopic (grain) and macroscopic levels $[1,2]$. Consequently, it is essential to understand the mechanisms responsible for the creation and evolution of internal stresses (or internal elastic strains), because their influence on the mechanical performance of the material is generally important. These properties and the crystallographic texture explain the appearance and development of important internal stresses when an elastoplastic deformation is introduced. These stresses are termed intergranular or secondorder stresses and strongly depend on initial and induced crystallographic textures. The engineering consequences of second-order stresses with strong texture could be severe [3].

Optimization of metal forming processes therefore requires proper knowledge of the evolution of the material anisotropic elastoplastic behaviour during plastic deformation. The modelling of the plastic deformation of metallic polycrystal can be carried out by deductive methods based on strain mechanisms and scale transition methods such as self-consistent (SC) models [4-6]. The micromechanical modelling seems to be particularly well suited to describe material evolution during deformation processes. Polycrystal models are typically evaluated by their ability to simulate the mechanical behaviour. In the case of small deformations (typically $<10 \%$ ), the texture development is minimal (when slip modes are dominant) and cannot serve as a way of assessing the model predictions. However, the model can be evaluated on a much more specific micromechanical level using the technique of lattice strain characterization by diffraction techniques (X-ray or/and neutron). They provide the possibility to determine the elastic lattice strain in selected grain subsets within the 
polycrystal as a function of the applied load. Such results can be directly compared with model predictions of volume average elastic lattice strains in selected diffracting grain subsets. In this case, the different material parameters (hardening matrix, critical resolved shear stresses (CRSS)) can be found by fitting the macroscopic loading curves and then validated with diffraction results. This capacity to measure intergranular strains provides a relevant experimental tool for understanding how intergranular strains are generated. Moreover, diffraction data offer a rigorous test for the models at a microscopic level [7-9].

In opposition to cubic alloys, hexagonal materials, such as zirconium and titanium alloys (with a $c / a$ ratio $<1.633$ ), are characterized by a large variety of possible deformation systems: prismatic glide $\{1010\}\langle 11 \overline{2} 0\rangle$ is reported to be the main active deformation mode $[1,10,11]$. Pyramidal $\langle c+a\rangle$ slip $\{10 \overline{1} 1\}\langle 11 \overline{2} 3\rangle$, basal slip $\{0002\}\langle 11 \overline{2} 0\rangle$ and pyramidal $\langle a\rangle\{10 \overline{1} 1\}\langle 11 \overline{2} 0\rangle$ are generally presented as secondary slip modes [11-14] more or less activated according to grain size, crystal orientation and material composition. At room temperature, twinning has also been observed in titanium samples [15-17]. Twinning of $\{10 \overline{1} 2\}$ and $\{11 \overline{2} 1\}$ types are expected in extension along the $\langle c\rangle$ axis, whereas twinning of $\{11 \overline{2} 2\}$ and $\{10 \overline{1} 1\}$ types are expected in compression along the $\langle c\rangle$ axis. Plastic deformation of titanium alloys is accommodated by a complex mixture of crystallographic slip and deformation twinning. It is difficult to identify the role played by the different deformation mechanisms in the overall behaviour. It is necessary to know both texture and deformation mechanisms to be able to model these mechanical properties. This requires, in particular, a proper knowledge of the deformation mechanisms with their corresponding CRSS. Few data on CRSS are available in the literature. Moreover, the CRSS generally depend significantly on the contents of alloying elements. It is still unclear which are the deformation systems actually activated in a polycrystal during straining.

In the present work, a complete study concerning the development and the evolution of internal strains under mechanical loading in a polycrystalline textured alpha titanium was made. Neutron diffraction techniques (constant wavelength and time-of-flight) in combination with a modified elastoplastic self-consistent (EPSC) model were used to interpret the lattice strain development and to deduce the main active deformation modes during uniaxial compression down to $-8 \%$. To understand how internal strains evolve in several directions for textured material, it is necessary to obtain a large data set concerning the behaviour of a grain group from a representative volume of the material under loading conditions. Measurements of lattice strains in directions both parallel and transverse to the loading axis were made. To characterize with accuracy the plastic anisotropy at the mesoscopic level, diffraction data were collected over 15 different $\{h k . l\}$ reflections at various load levels. This provided a comprehensive data set to test the scale transition model developed for this study.
A modification concerning the selection of active slip systems was made for the present model, and a different formulation of the crystal plasticity was applied. This algorithm is much faster and resolves the problem of ambiguous selection of slip systems. The theoretical strains are compared with the experimental results obtained by neutron diffraction. The results predicted by the SC model are discussed. In particular, twinning mode is treated as a directional deformation system, and the reorientation by slip and twinning is taken into account in the calculations.

\section{Experimental details}

\subsection{Experimental procedure}

The material investigated is a commercially pure $\alpha$-titanium (CP-Ti). Its chemical composition is $\mathrm{C}(0.07)$, $\mathrm{N}(0.008), \mathrm{O}(0.12), \mathrm{H}(0.0013)$, balance Ti (wt.\%). Conventional metallography revealed a nearly equiaxed grain size of $50 \mu \mathrm{m}$. Compression samples were taken from the parent bar. The samples were $8 \mathrm{~mm}$ in diameter and $14 \mathrm{~mm}$ in height. They were tested using the SALSA monochromatic neutron diffraction strain scanning instrument at the ILL neutron source in Grenoble, France (incident beam wavelength $1.648 \AA$ ) [18]. A two-dimensional position-sensitive detector with an angular opening of $\sim 5^{\circ}$ is typically mounted on the SALSA. A horizontal load frame with a $50 \mathrm{kN}$ capacity is used to perform in situ measurements of lattice strains during uniaxial compression. The system was placed on the sample table, a six-axis robotic Stewart platform (hexapod). The load frame was rotated in such a way that the loading axis was parallel to the scattering vector, i.e., bisecting the angle between the incident and the diffracted beam, making available the measurement of lattice strains in the loading direction (denoted LD). A gauge volume of $\sim 3 \times 3 \times 3 \mathrm{~mm}^{3}$ including beam divergence was defined by the primary and secondary instrument slits. The macroscopic strain was determined concurrently using an extensometer that spanned the irradiated region. Raw data were analysed using the LAMP software [19] allowing the peak parameters to be obtained for each reflection. In particular, peak positions were determined by fitting the experimental data by a Gaussian function with a linear background.

Additional neutron diffraction measurements were performed on the ENGIN-X beamline at the ISIS facility, Rutherford Appleton Laboratory (Didcot, UK) [20], using a $50 \mathrm{kN}$ Instron testing machine. Briefly, the ENGIN-X instrument presents a horizontal loading axis positioned at $45^{\circ}$ to the incident beam. Two detector banks are set up horizontally and at angles $\pm 90^{\circ}$ to the incident beam, allowing simultaneous measurements of lattice strains in both parallel (LD) and perpendicular (transverse direction, TD) directions to the applied load, in the opposing $90^{\circ}$ detector banks (Fig. 1). The time-of-flight technique enables collection of an entire diffraction pattern (effective $d$-spacing range from 0.88 to $2.63 \AA$ ) in each detector 
simultaneously, owing to the specificity of the neutron beam. The detector banks cover $\pm 14^{\circ}$ in the horizontal plane and $\pm 21^{\circ}$ in the vertical plane [20].

The gauge volume was $4 \times 4 \times 4 \mathrm{~mm}^{3}$, and the recording time was taken over $43 \mathrm{~min}$ for each measurement at a given macroscopic strain. Macroscopic strain was monitored on the sample using a clip gauge. Single peak fitting was performed using the Open Genie code [21].

A series of increasing uniaxial compressive loads were applied. To avoid the strain relaxation owing to the creep behaviour at room temperature, in the plastic regime, samples were first deformed to the desired macroscopic strain and then immediately unloaded, studying just the residual strains remaining after a given increment of plastic deformation, at zero applied load (Fig. 2).

For each peak analysed, the elastic strain $\langle\varepsilon(h k . l)\rangle_{V_{d}}$ of a group of grains with a common $\{h k . l\}$ plane-normal parallel to the diffraction vector can be found from the measured lattice spacing $\langle d(h k . l)\rangle_{V_{d}}$ and a reference lattice spacing $d_{0}(h k . l)$ using the following expression:

$\langle\varepsilon(h k . l)\rangle_{V_{d}}=\frac{\langle d(h k . l)\rangle_{V_{d}}-d_{0}(h k . l)}{d_{0}(h k . l)}$

The reference point for this calculation is the initial measured $d_{0}(h k . l)$ for each lattice plane before deformation of the material. \langle\rangle$_{V \mathrm{~d}}$ is the average over diffracting grains for the $\{h k . l\}$ reflection considered. Lattice strains presented in this work are given as micro-strain $(\mu \varepsilon$, units of $10^{-6}$ ).

One should notice that the lattice spacing corresponding to each measured $\{h k . l\}$ diffraction peak represents a distribution of interplanar spacings of $\{h k . l\}$ planes within a subset of grains having a $\{h k . l\}$ plane-normal parallel to the diffraction direction, either the LD (denoted $\{h k . l\}_{/ /}$) or the TD (denoted $\{h k . l\}_{\perp}$ ). Eq. (1) allows the determination of an average elastic strain within this set of grains (and not within an individual grain or orientation). The error bars on the different plots reflect experimental uncertainties in establishing the diffraction peak positions.

Strains measurements were performed on 15 plane families: $\{10.0\},\{00.2\},\{10.1\},\{10.2\},\{11.0\},\{10.3\},\{11.2\}$,

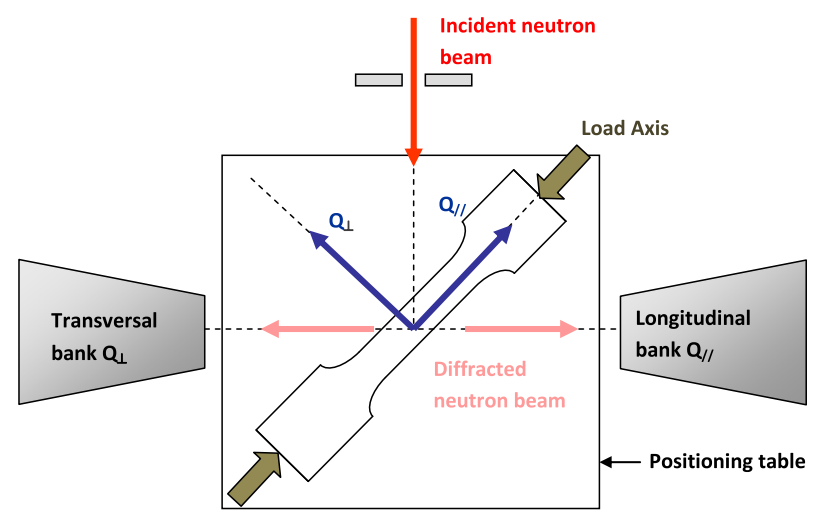

Fig. 1. Schematic view of the neutron diffraction instrument ENGIN-X (ISIS facility, UK).

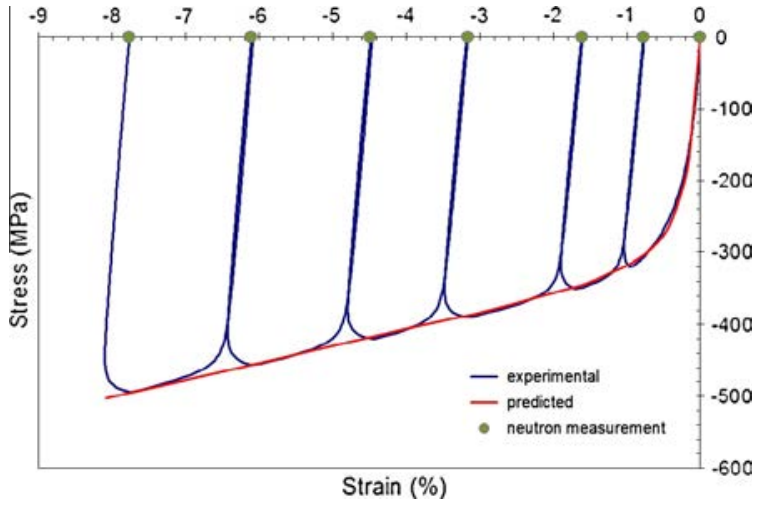

Fig. 2. Comparison between modelled and measured macroscopic stressstrain curves. Points indicate average strain during neutron measurement (with ENGIN-X) after unloading at zero applied load.

$\{20.1\},\{20.3\},\{21.0\},\{21.1\},\{11.4\},\{21.2\},\{10.4\}$, $\{10.5\}$. Only results from first-order peaks are reported. The $\{20.0\},\{00.4\}$ and $\{20.2\}$ measured lattice strains are the same as those of the $\{10.0\},\{00.2\}$ and $\{10.1\}$, respectively, with lower intensity, and thus are not reported in the following because of the initial texture, the set of peaks is not the same for the two detector banks (Fig. 6).

\subsection{Texture development: activity of tensile twinning}

Texture measurements were performed with the GEM beamline at the ISIS facility. Detailed information about the instrument and the technique used to determine the texture can be found in Refs. [22] and [23]. Fig. 3a shows the initial texture. The samples have a strong asymmetric texture in the ND-TD (ND, normal direction) plane with the majority of grains with their basal normal close to the TD and perpendicular to the loading axis (LD). The experimental $\{00.2\}$ and $\{10.0\}$ pole figures (PF) are shown in Fig. $3 b$ after $-6 \%$ deformation. One could observe the formation of a basal component along the straining direction. This reorientation of the $\{00.2\}$ poles from the TD to the straining direction is associated with deformation twinning. Actually, the activation of tensile twinning produces a $\sim 85^{\circ}$ reorientation of the basal poles [24]. Therefore, compressive loading along the LD with the $\langle c\rangle$ axis close to the TD reorients the $\langle c\rangle$ axis of the grains undergoing deformation twinning towards the LD. In order to quantitatively determine the twin volume fraction, the difference $\{002\}$ PF between the as-received sample and that loaded to a total of $-6 \%$ strain may be integrated over a sub-space associated with the twin component [25]. Based on the previous observations obtained in Fig. 3, an area within $35^{\circ}$ of the straining direction was chosen. Then, the total twin volume fraction after $-6 \%$ strain is 0.16 .

The instrument geometry (associated with the time-offlight beam) used with the strong basal texture and sample orientation is particularly suitable for investigating the propagation of deformation tensile twinning. It was shown for $\mathrm{Mg}$ and $\mathrm{Zr}$ alloys [26,27] that there is a direct correlation between the measured diffraction intensity variations 

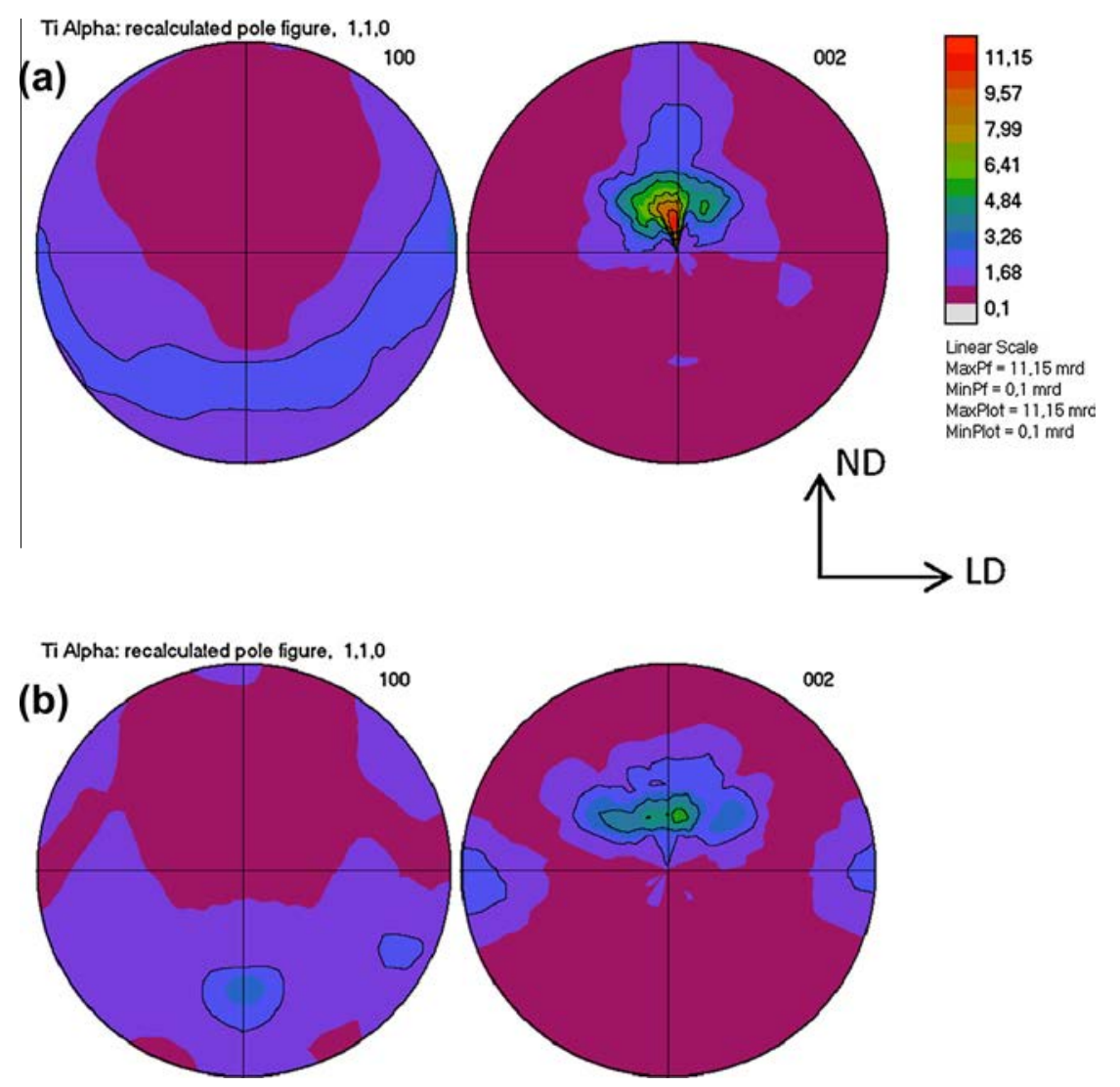

Fig. 3. Texture of Ti sample (a) initially and (b) after a $-6 \%$ compression: the experimental $\{00.2\}$ and $\{10.1\}$ PF are shown the ND-LD plane, the center of the poles being the TD.

and the appearance of twins. Owing to the texture of the as-received sample, the initial intensity of the $\{00.2\}_{\perp}$ peak is higher in the transversal detector $Q_{\perp}$, while the $\{10.0\}_{\perp}$ peak intensity has a lower intensity (Fig. 4). In contrast, the $\{002\} / /$ reflection is quasi-absent in the initial longitudinal diffraction pattern, unlike the diffraction peaks from prism planes $\{10.0\}_{/ /}$and $(11.0\} / /$. With macroscopic deformation, as shown in Fig. 4, an increase in the $\{00.2\} / /$ diffraction peak intensity is observed with a concurrent decrease in the $\{10.0\} / /$ peak; this reflects a change in the grain volume fraction diffracting into the axial detector bank. The opposite behaviour is observed in the transversal detector. The tensile twinning mode results in an exchange of $\{00.2\}$ peak intensity from the transversal (corresponding to the parent grains, i.e., grains undergoing twinning) to the longitudinal detector bank (corresponding to the newly twin grain families) with a nearly $90^{\circ}$ reorientation of the crystal lattice, which can be seen owing to the wide acceptance of the detectors. Fig. 5 shows the relative intensity changes in different diffraction peaks in the $Q_{/ /}$and $Q_{\perp}$ detectors as a function of the applied compressive strain. To detect the onset of the twinning process, it can be easy to observe the intensity changes with $\{k k . l\}$ orientations that are initially weak, i.e., the $\{00.2\}_{/ /}$and $\{10.0\}_{\perp}$ grains corresponding to the newly twin grains. It is clear that the intensity remains approximately constant down to a total of $-1.5 \%$ total strain for both $\{00.2\}_{/ /}$and $\{10.0\}_{\perp}$.
Beyond this strain value, the $\{00.2\}_{/ /}$and $\{10.0\}_{\perp}$ intensities increase significantly, pointing out the onset of twinning. For $\{h k . l\}$ reflections corresponding to the parent grain families, $\{10.0\}_{/ /},\{20.1\}_{/ /},\{11.0\}_{/ /},\{21.1\}_{/ /}$, the intensity evolution is naturally opposite to what is observed in twin grain orientations. For these reflections, the onset of twinning is difficult to discern. The initial intensity changes are less obvious. When the twinning mode is activated, only a small fraction of the parent grains are concerned at the beginning and it is difficult to observe precisely such a small reduction in peak intensity from prism planes associated with higher initial diffraction intensity (Fig. 4). Nevertheless, it could be noted that the $\{20.1\} / /$ intensity decreased significantly at the same rate as the $\{10.0\}_{/ /}$reflection, followed by the $\{21.1\}_{/ /}$and $\{11.0\}_{/ /}$orientations. This reflects clearly some twinning activity in these grains, which are favourably oriented for the tensile twinning mode (the Schmid factors are: $\quad m_{\{10.0\} / /}=0.5, \quad m_{\{20.1\} / /}=0.45, \quad m_{\{21.1\} / /}=0.42$, $\left.m_{\{11.0\} / /}=0.39\right)$.

\section{Self-consistent modelling}

\subsection{Model description}

The principles for using the SC model to predict elastoplastic deformation were proposed by Kröner and Hill 

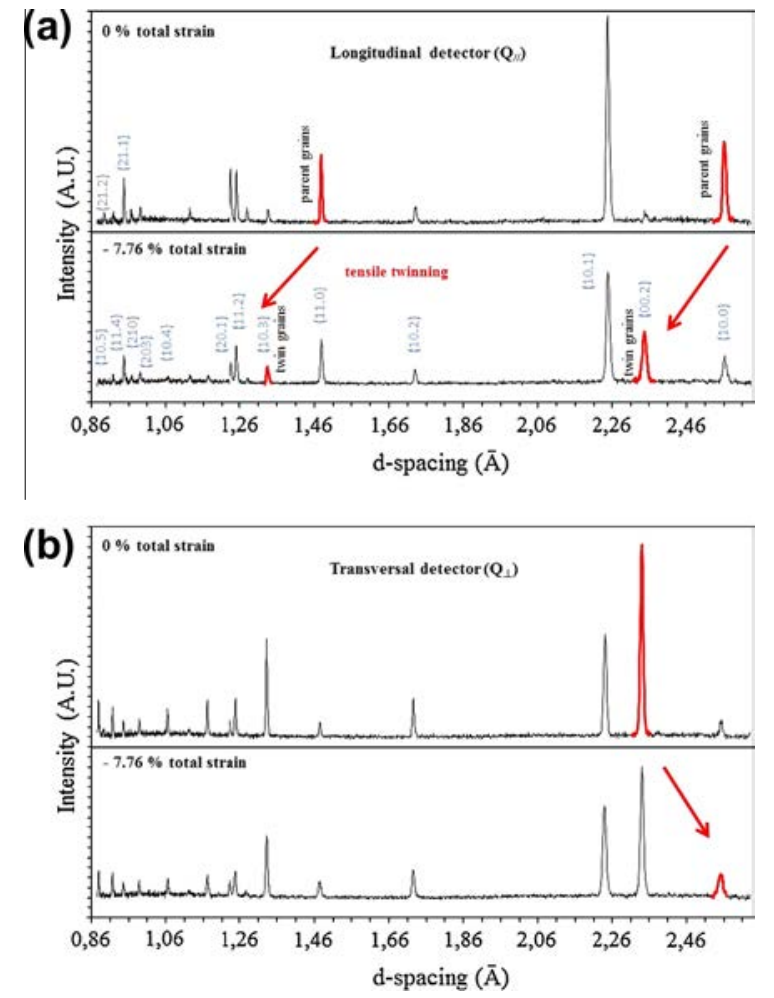

Fig. 4. Neutron diffraction patterns obtained in the (a) longitudinal $Q_{/ /}$ and (b) transversal $Q_{\perp}$ detectors from ENGIN-X. The red peaks show the evolution of the intensity between the parent and the twin grains for some particular orientations linked to the tensile twinning during the compression tests. (For interpretation of the references to colour in this figure legend, the reader is referred to the web version of this article.)

$[28,29]$. In the present work, the model developed in Refs. [4] and [30] is used. Refs. [31] and [32] present a more detailed description of the EPSC model. Each grain is assumed to have the form of an ellipsoidal inclusion in a homogeneous effective medium, whose properties are the average of all the other grains in the assembly. The measured initial texture was used as the input to the EPSC model: the polycrystal is represented by a weighted discrete distribution (2100 grains) of orientations (Euler angles $\left.\left(\varphi_{1}, \phi, \varphi_{2}\right)\right)$. Lattice rotations (reorientation by slip and twinning) and texture changes are included in the model.

The principal modification, which is briefly discussed here, concerns the selection of active slip systems. This algorithm is much faster and resolves the problem of the ambiguous selection of slip systems. The plastic flow can take place in a grain when the Schmid criterion is verified, i.e., slip (or twinning) occurs if the resolved shear stress $\tau_{g}$ on a system $\mathrm{g}$ is equal to the critical value $\tau_{c}^{g}$, depending on the hardening state of the slip system. This condition is necessary, but is insufficient. Thus, the complementary condition stating that the increment of the resolved shear stress must be equal to the incremental rate of the CRSS has to be checked simultaneously. The resolved shear stress is defined as the projection of the mesoscopic stress tensor $\sigma^{I I}$ on the considered deformation system. In small strain formulation, one has
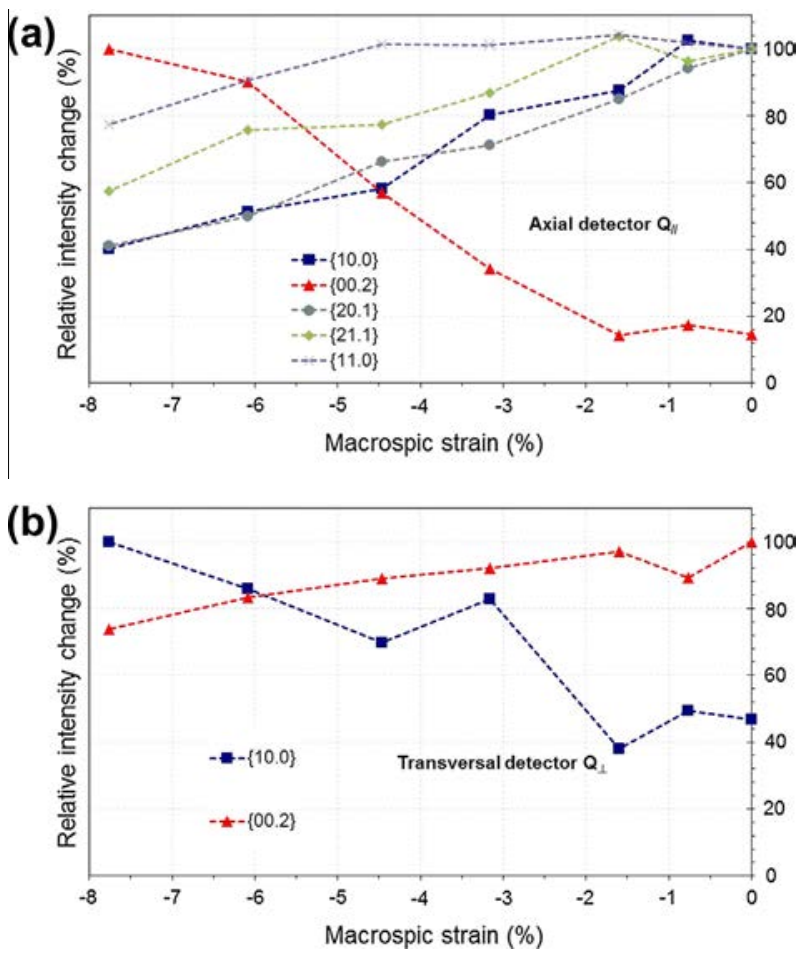

Fig. 5. Evolution of the integrated intensities of several neutron diffraction measured peaks in the (a) longitudinal and (b) transversal ENGIN-X detectors at ISIS facility. The different intensities are normalized by the initial value for the disappearing peaks and by the final value for the appearing peaks. The standard deviation on the experimental intensities is $<10 \%$. The lines serve as a guide to the eye.

$\tau=R^{g}: \sigma^{I I}=\tau_{c}^{g}$ and $\dot{\tau}^{g}=R^{g}: \dot{\sigma}^{I I}=\dot{\tau}_{c}^{g}$

where $R^{g}$ is the Schmid tensor on a system g. $A: B$ denotes the double scalar product $A_{i j k l} B_{k l m n}$ using the Einstein summation convention.

The main problem is to determine which combination of deformation systems will actually be activated at each step of the plastic deformation path. Therefore, all possible combinations of potentially active systems must be scanned to find one that satisfies the two previous conditions (Eq. (2)) simultaneously. Because this must be done in a large deformation framework, running time considerations become one of the main problems of the model. Moreover, this method can give several equivalent solutions for some hardening matrices [33]. Franz et al. [34,35] proposed a new formulation to determine the slip system activity in the elastoplastic regime. The accuracy of the simulations was also evaluated at the meso- and macroscopic levels by referring to mechanical experiments (tensile tests, neutron diffraction) [36]. It should be noticed that the "classic" EPSC model and the modified one give similar results: the main active systems, predicted texture and residual strains are similar. Based on Refs. [34] and [35], the slip rate on a system $\mathrm{g}$ can be expressed by the following equation:

$\dot{\gamma}^{g}=M^{g}\left(\tau^{g}, \tau_{c}^{g}\right) \dot{\tau}^{g}$ 
The slip rate is linked to the resolved shear stress rate through a function $M^{g}$.

The hardening parameter $M^{g}$ is given by [34]

$$
\begin{aligned}
M^{g}= & \frac{1}{H^{g g}}\left[\frac{1}{2}\left(1+\operatorname{th}\left(k_{0}\left(\frac{\tau^{g}}{\tau_{c}^{g}}-1\right)\right)\right)\right]\left[\frac{1}{2}\left(1+\operatorname{th}\left(\dot{\tau}^{g}\right)\right)\right] \\
& \times\left[\frac{1}{2}\left(1+\operatorname{th}\left(\tau^{g}\right)\right)\right]
\end{aligned}
$$

where $k_{0}$ is a numerical parameter. $H^{g g}$ is the self-hardening parameter defined in Eq. (10). The hyperbolic tangent function was tested and used because it allows the mechanical and hardening behaviours to be reproduced. The slip rate can be written as

$\dot{\gamma}^{g}=M^{g} \dot{\tau}^{g}=M^{g} R^{g}: \dot{\sigma}^{I I}$ (without summation over $\mathrm{g}$ )

If an additive decomposition of strain rate $\dot{\varepsilon}^{I I}$ on elastic and plastic parts is used:

$\dot{\varepsilon}^{I I}=\dot{\varepsilon}^{I l e}+\dot{\varepsilon}^{I I D}$

Using the usual generalized Hooke's law and Eq. (5), the slip rate on a system $\mathrm{g}$ becomes

$\dot{\gamma}^{g}=M^{g} R^{g}:\left(c: \dot{\varepsilon}^{I l e}\right)$

The plastic strain rate $\dot{\varepsilon}^{I l p}$ is related to the tensor $R^{g}$ and the slip rate $\dot{\gamma}^{g}$ in system $\mathrm{g}$ by the expression

$\dot{\varepsilon}^{p}=\sum_{g} R^{g} \dot{\gamma}^{g}$

With Eqs. (6)-(8), one obtains, after calculations, the following relation:

$\dot{\gamma}^{g}=\sum_{h}\left(\delta_{g h}+M^{g} R^{g}: c: R^{h}\right)^{-1}: M^{h}: R^{h}: c: \dot{\varepsilon}^{I I}$

It is usual to introduce a hardening matrix $H^{g h}$ to describe the evolution of CRSS rate in a system $\mathrm{g}$ as a function of the plastic slip (or twinning) on the other systems:

$\dot{\tau}_{c}^{g}=\sum_{h} H^{g h} \dot{\gamma}^{h}$

With relations (6), (9) and (10) and the usual generalized Hooke's law, the constitutive elastoplastic relationship for the single crystal can be deduced:

$$
\begin{aligned}
\dot{\sigma}^{I I} & =\left[c-\sum_{g} \sum_{h} c: R^{g}\left(\delta^{g h}+M^{g} R^{g}: c: R^{h}\right)^{-1} M^{h} R^{h}: c\right] \\
& : \varepsilon^{I}=1: \dot{\varepsilon}^{I I}
\end{aligned}
$$

where $l$ is the tensor of the elastoplastic tangent moduli of the single crystal.

The relation between the stress rate $\dot{\sigma}^{I}$ and the strain rate $\dot{\varepsilon}^{I}$ can be written for the macro-scale as follows:

$\dot{\sigma}^{I}=L: \dot{\varepsilon}^{I}$

where $L$ is the macroscopic tangent modulus for the fictional average homogeneous medium. The local strain and stress rates can be obtained classically through the localization $A$ and concentration $B$ tensors:

$$
\begin{aligned}
\dot{\varepsilon}^{I I} & =\left(I+S^{e s h}: L^{-1}: \Delta l\right)^{-1}: \dot{\varepsilon}^{I}=A: \dot{\varepsilon}^{I} \\
\dot{\sigma}^{I I} & =l:\left(I+S^{e s h}: L^{-1}: \Delta l\right)^{-1}: L^{-1}: \dot{\sigma}^{I}=l: A: L^{-1} \\
& : \dot{\sigma}^{I}=B: \dot{\sigma}^{I}
\end{aligned}
$$

where $\Delta l=l-L . S^{e s h}$ is the Eshelby tensor.

The volume averages of the local stress and strain tensors must coincide with the overall strain and stress. After some algebraic calculations, these conditions give the overall elastoplastic tensor $L$ as a weighted average of the mesoscopic tensor $l$ :

$L=\left\langle l:\left[I+S^{e s h}: L^{-1}: \Delta l\right]^{-1}\right\rangle$

Eq. (15) is a non-linear implicit equation, because $S^{\text {esh }}$ depends on the unknown $L$ in the framework of EPSC theory. The Eshelby tensor is calculated by an integral equation [31], which takes the plastic anisotropy fully into account. This equation is solved iteratively.

Concerning the incorporation of twinning, the current EPSC approach uses the same condition for twin activation as for slip activation. Assuming that the twinning plane normal vector and the shear direction vector corresponding to the twinning are equivalent to the slip plane normal and the slip direction vectors, respectively, they are incorporated into the plasticity model. The shear strain rate induced by twinning is calculated in the same manner as that for slip deformation. When twin systems become active, the orientation of the twinned grain is calculated from the crystallographic relationship between the parent and the twin. The effective volume fraction for a twin system $\mathrm{g}$ for a grain is given by:

$f_{\text {twin }}=f_{i} \frac{\dot{\gamma}^{g}}{S}$

where $S$ is the characteristic twin shear, and $\dot{\gamma}^{g}$ is the shear strain for the twin system g. $f_{i}$ is the weight of the grain I, which corresponds to the volume fraction of the grain in the aggregate. When twinning is active, the fraction of the grain that reorients is transferred to the corresponding grain that has the closet orientation in Euler space and added to the fraction already assigned to this grain. The number of grains is kept unchanged throughout the analysis. It should be noticed that 2100 orientations with suitable weights were chosen randomly from the experimental ODF. No region in orientation space is totally ignored with this discretization technique.

This twinning model does not account for the local stress relaxation due to twinning. It only can provide some degree of stress relaxation in the parent grains. This point is discussed in Section 4.

Once $L$ is known, by specifying an overall stress or strain increment, the rate of strain at the grain level $\dot{\varepsilon}^{I I}$ can be determined, and other variables characterizing grain evolution and depending on $\dot{\varepsilon}^{I I}$ can be calculated. The 
calculations are performed up to a given total strain $\varepsilon^{I}$ and then, an elastic unloading of the sample is performed in the last step $\left(\sigma^{I} \rightarrow 0\right)$. The mesoscopic elastic strain tensor $\varepsilon^{I l e}$ is determined for each grain when $\sigma^{I}=0$. Then, $\left\langle\varepsilon(h k . l)_{V_{d}}\right\rangle$ is calculated for each analysed plane and compared with experimental data obtained by neutron diffraction. A subset of the total population of grains used in the EPSC model is identified for each diffracting family defined by the condition of having an $(h k . l)$ plane lying within $\pm 14^{\circ}$ (corresponding to the horizontal plane defined in Section 2.1) and $\pm 21^{\circ}$ (i.e., the vertical plane) of the loading axis or perpendicular to it. These are the grains that would contribute to a diffraction measurement of strain in that direction, and an average over each subset of the strains across the diffracting plane is compared with the neutron results.

\subsection{Data used in the simulations}

The single crystal elastic constants used in this model are: $c_{11}=c_{22}=162.4 \mathrm{GPa}, \quad c_{12}=92 \mathrm{GPa}, c_{13}=69 \mathrm{GPa}$ and $c_{44}=46.7 \mathrm{GPa}$ [37]. The $k_{0}$ value (Eq. (4)) is determined by comparing with a "classic" EPSC model under monotonic deformation (in terms of main active deformations systems, crystal reorientation). For all simulations, $k_{0}$ is equal to 25. The initial CRSS are taken as identical for all systems of a system type. The deformation systems introduced in the model are assumed to be: prismatic slip denoted $\operatorname{pr}\langle a\rangle$, pyramidal slip $(\operatorname{pyr}\langle c+a\rangle$ and $\operatorname{pyr}\langle a\rangle)$, basal slip (bas $\langle a\rangle$ ) and $\{10 \overline{1} 2\}$ twinning (ttw). Without assumption, there are a large number of material parameters, five CRSS and $841(=29 \times 29)$ elements in the $H^{g h}$ matrix (Eq. (10)), that need to be specified.

In order to obtain a tractable description of the crystal hardening (and to explore the feasible parameters), the following simple form is assumed for the hardening matrix: $H^{g h}=q H^{h h}$. Owing to a lack of relevant data on the nature of latent hardening in titanium alloys, the interactions between different deformation modes are described by a very simple law. The factor $q$ determines the degree of latent hardening: $q=0$ provides only self-hardening, and $q>1$ provides stronger latent hardening than self-hardening. $q$ is taken to be constant and fixed to a value of $q=1.3$ for the simulations. In the present calculation, the constant value of $H^{g g}$ (for a given deformation mode g) and $q$ assumed for the plastic deformation lead to the linear hardening law.

This study uses a single set of material parameters to describe the entire data set (the macroscopic stress-strain curve and the residual elastic strains measured for the different grain families) under compression and all in two principal directions.

The macroscopic yield strength and the curvature of the elastic-plastic transition during compression tests was used as the fitting criteria to determine the value of the CRSS and the self-hardening for $\operatorname{pr}\langle a\rangle$. Based on the experimental results obtained in Section 2.2, the values for the twin hardening parameter were established by fitting the measured twin volume fraction experimentally found at $-6 \%$ macroscopic strain, and the value of CRSS is chosen to activate tensile twinning at around $-1.5 \%$ total strain.

Concerning $\operatorname{pyr}\langle c+a\rangle, \operatorname{pyr}\langle a\rangle$ and bas $\langle a\rangle$, it is difficult to identify, a priori, the role played by the different deformation mechanisms in the overall behaviour. The lack of sufficient knowledge regarding values of hardening parameters appropriate for deformation modes constitutes a difficulty for performing polycrystalline modelling. To circumvent this difficulty, each deformation mode was applied independently within the EPSC model, in order to examine its effect on the strain accumulation. Based on the previous analysis, $\operatorname{pr}\langle a\rangle$ and ttw were naturally invoked in all the performed simulations. The effect of combining two and three systems was also examined with different sets of material parameters (CRSS, hardening matrix) to give optimum agreement between the measured and predicted results. Finally, the best agreement was found with the values given in Table 1 .

\section{Results and discussion}

Fig. 2 shows the comparison between the measured and predicted stress-strain curves in compression. The experimental yield stress is $\sim-195 \mathrm{MPa}(-0.19 \%$ total strain). The predicted and measured plastic slopes are identical down to $-8 \%$. The calculated Young's modulus (106 GPa) coincides well with the experimental one $(104 \pm 3 \mathrm{GPa})$. The model captures the observed flow curve with excellent agreement.

The development of the elastic strains in different grain orientations can be used as a useful and sensitive sensor of plasticity. Before discussion of the results, it may be useful to remind the origin of the residual intergranular strains $[27,38]$. When a polycrystalline aggregate is submitted to a uniaxial loading, in the elastic region, the lattice strains develop linearly with load up to the point of micro yielding. Owing to their inherent elastic anisotropy and their orientation, there is a difference in the elastic response for each grain family (i.e., all grains with a particular $\{h k i l\}$ planes perpendicular to the scattering vector $Q$ for the given detector bank). When plastic deformation occurs, slip activity is initiated in certain grains similarly orientated. The imposed deformation is plastically accommodated, and these grains carry less load. In other words, the external load is redistributed from plastically softer grain families towards plastically harder grain families.

\section{Table 1}

Material parameters used in the simulations.

\begin{tabular}{lllll}
\hline$\tau^{\mathrm{pr}\langle\mathrm{a}\rangle}(\mathrm{MPa})$ & $\tau^{\mathrm{pyr}\langle\mathrm{a}\rangle}(\mathrm{MPa})$ & $\tau^{\mathrm{bas}\langle\mathrm{a}\rangle}(\mathrm{MPa})$ & $\tau^{\mathrm{pyr}\langle\mathrm{c}+\mathrm{a}\rangle}(\mathrm{MPa})$ & $\tau^{\mathrm{ttw}}(\mathrm{MPa})$ \\
\hline 68 & 120 & 175 & 250 & 230 \\
$H^{\mathrm{pr}\langle\mathrm{a}\rangle}$ & $H^{\mathrm{pyr}\langle\mathrm{a}\rangle}$ & $H^{\mathrm{bas}\langle\mathrm{a}\rangle}$ & $H^{\mathrm{pyr}\langle\mathrm{c}+\mathrm{a}\rangle}$ & $H^{\mathrm{tw}}$ \\
$(\mathrm{MPa})$ & $(\mathrm{MPa})$ & $(\mathrm{MPa})$ & $(\mathrm{MPa})$ & $(\mathrm{MPa})$ \\
\hline 142 & 175 & 200 & 300 & 220 \\
\hline
\end{tabular}


This behaviour results in a deviation in the lattice strain linear response to the applied load. With increasing load, the lattice strain evolution is characterized by several inflexions, reflecting the changes in the load shared among diverse orientations as different deformation modes activate. At the end of the test, the release of the applied load gives rise to residual lattice strains as shown in Fig. 6. The development of the longitudinal (transversal) elastic strains after unloading is shown in Fig. 6a (Fig. 6b) for all the studied $\{h k . l\}$ reflections. These neutron measurements clearly show the effective existence of plastic anisotropy. As Eq. (3) shows, the analysed lattice strain depends on a function of the analysed planes family. Strain incompatibilities are present at the mesoscopic level in the material. Consequently, the strains obtained by neutron diffraction depend on the plane considered. The diffracting crystals are not the same for each case, and this allows one to deduce that different and important intergranular strains exist, coupled with a strong plastic anisotropic deformation for these plane families.

The longitudinal lattice strains were measured using the ENGIN-X and SALSA spectrometers. For the transverse lattice strains, owing to the allocated neutron beam time limitations, the experiment was only performed on the ENGIN-X beamline at the ISIS facility.

Concerning the longitudinal lattice strains, similar values are observed between the two measured data sets except for $\{11.2\} / /$. With the TOF diffraction measurements, compressive residual intergranular strains $(\sim-500 \mu \varepsilon)$ are observed, whereas the $\{11.2\} / /$ orientation, with the SALSA beamline shows residual strain values ranging from -31 to $-131 \mu \varepsilon$. Despite this difference, a similar trend is observed for this orientation, which accumulates a compressive strain. In this particular case, the diffraction averaging over different detector areas on ENGIN-X $\left(28 \times 42^{\circ}\right.$ ENGIN-X detector windows $)$ and on SALSA $\left(5 \times 5^{\circ}\right)$ does not appear to be a significant factor in the evolution of lattice strain.

There is an interesting observation of the experiment about the $\{00.2\} / /$ orientation. This peak develops significant compressive residual lattice strain $(\sim-2000 \mu \varepsilon)$ during the first $-1 \%$ of total strain. This indicates that the $\{00.2\} / /$ grains support a larger fraction of each stress increment after plasticity. This is expected, since these grains are poorly orientated for the soft $\langle a\rangle$ slip modes. Similar behaviour can be seen in $\{10.3\} / /,\{11.4\}_{/ /}$and $\{10.2\} / /$ orientations, although the lattice strains are smaller: -1500 , -1100 and $-875 \mu \varepsilon$, respectively. These grain families correspond to orientations measured nearest to the $\{00.2\}_{/ /}$ one (the angles from basal plane are equal to $31^{\circ}, 42^{\circ}$ and $42^{\circ}$, respectively).

Fig. 6a shows that, after compressive loading, significant positive residual strains develop in the $\{10.0\}_{/ /}$and $\{21.0\}_{/ /}$ grains $(765 \mu \varepsilon$ and $1000 \mu \varepsilon$, respectively, at $-7.75 \%$ of total strain). A similar trend, with smaller strain values, is observed for the $\{20.1\}_{/ /}(535 \mu \varepsilon),\{11.0\}_{/ /}(372 \mu \varepsilon)$ and $\{21.1\} / /(255 \mu \varepsilon)$ orientations.
Fig. 6 shows the measured and modelled lattice response in grains with $\{h k . l\}$ plane-normal parallel and perpendicular to the applied stress in compression. Fig. 6a shows that the model predictions of the residual strains parallel to the tensile axis are fairly accurate for the 13 studied reflections in terms of trend and magnitude. The use of the EPSC model allows an explanation of the origin of these residual strains.

In the simulations, the $\{10.0\} / /,\{11.0\} / /$ and $\{21.0\} / /$ grains yield first at $-155 \mathrm{MPa}$, linked to the activation of prismatic slip, which has the lowest CRSS with the highest Schmid factor for these orientations. They undergo plastic deformation below the macroscopic yield stress ( $\sim 195 \mathrm{MPa})$. A significant decrease in their lattice strain is observed with a departure from linearity of the lattice strain response to the applied load. At the same time, a dramatic increase in the lattice strain of the $\{0002\}_{/ /},\{10.3\}_{/ /}$, $\{11.4\}_{/ /}$and $\{10.2\} / /$ orientations is detected in comparison with their initial linear slopes. This indicates that the grains of the $\{0002\}$ // family bear more stress, while the grains of the $\{10.0\}_{/ /},\{11.0\}_{/ /}$and $\{21.0\}_{/ /}$families bear relatively less stress. With further increase in applied stress, the load is then transferred to other unyielded orientations. This can be explained by the large prismatic activity and weak pyramidal $\langle c+a\rangle$ and/or twinning activities $<-1 \%$ strain. The former system accommodates deformation perpendicular to the $\langle c\rangle$ axis, and the latter systems accommodate deformation along the $\langle c\rangle$ axis. If pyramidal $\langle c+a\rangle$ and/or twinning are not active, more elastic strain accumulates along the $\langle c\rangle$ axis.

Focusing again on the $\{00.2\} / /$ grains, a pronounced strain relief is observed in these grain families (grains compressed parallel to their $\langle c\rangle$ axis) at a prestrain of $\sim-1 \%$ : the corresponding lattice strain increases from $\sim-2000 \mu \varepsilon$ to $\sim-1150 \mu \varepsilon$ at $\sim-3 \%$ total strain. This sudden lattice strain relief in the $\{00.2\} / /$ grains goes with an increase in the $\{00.2\} / /$ diffraction peak intensity and a concurrent decrease in the $\{10.0\}_{/ /}$diffraction peak intensity (see Section 2.2). These observations signal the onset of tensile twinning. The strain measured by neutron diffraction is an average over the initially present $\{00.2\} / /$ grains and the newly twin grains. This reduction in the magnitude of the compressive strain can be explained by the fact that the twin grains contribute mainly to the diffraction peak response, owing to the lack of initial $\{00.2\} / /$ grains. These newly reoriented grains with lower lattice strain begin to contribute to the neutron averaging and reverse the average $\{00.2\} / /$ strain.

It should be noted at this point that the twinning mode is treated in the model in the same way as plastic slip, with twins characterized by their plane, direction with a CRSS and a hardening description. The present model is only rigorously applicable if slip is the main deformation mode. It is inappropriate for cases where twinning accommodates a major fraction of the strain.

The present approach includes only the reorientation of the twinned domain and its effect on texture, but it does not 
(a)
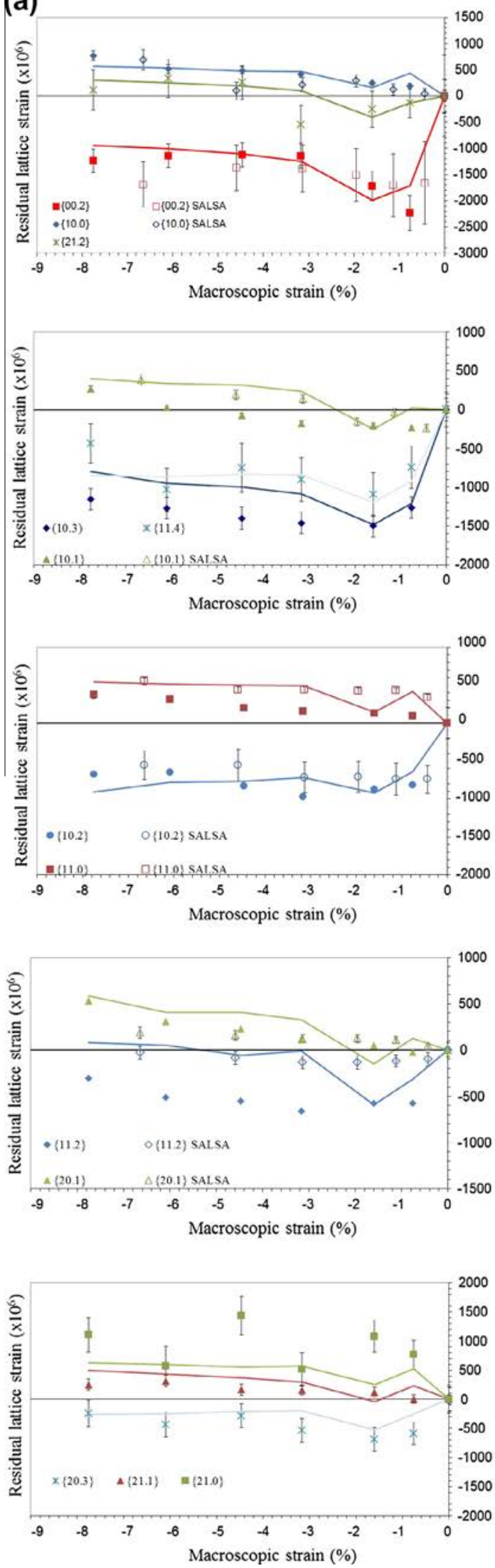

(b)
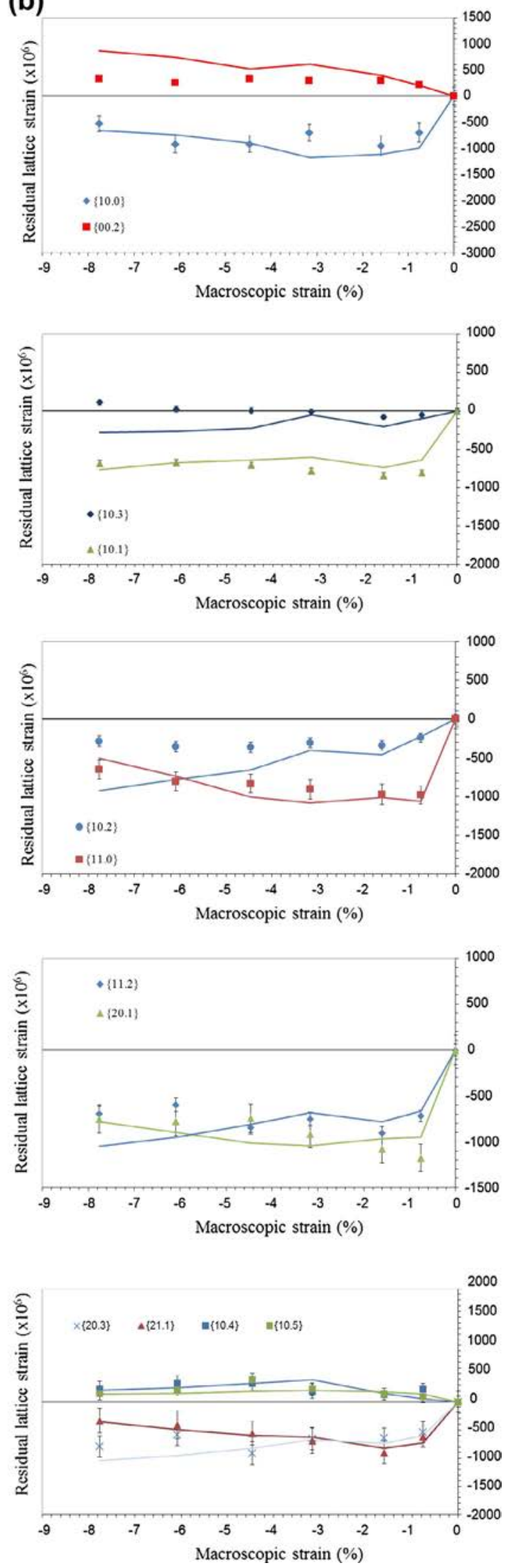

Fig. 6. Residual lattice strains associated as a function of the total strain with different $\{h k . l\}$ reflections (a) parallel and (b) perpendicular to the applied compression. Closed (open) symbols represent the experimental results obtained at the ENGIN-X (SALSA) neutron beam line. The coloured lines represent the model predictions. (For interpretation of the references to colour in this figure legend, the reader is referred to the web version of this article.) 
take into account stress relaxation and the resulting stress state of the twin or any changes in constraint due to the presence of a twin boundary. Despite this simplistic approach, the model provides further relaxation modes in the parent grains.

The model predicts the yielding of $\{10.2\} / /$ grains at $-258 \mathrm{MPa}(-0.4 \%$ total strain) induced by the activation of bas $\langle a\rangle$ slip. With higher macroscopic stress, $\operatorname{pyr}\langle a\rangle$ has significant activity. The $\{10.3\} / /$ grains are predicted to yield at $-290 \mathrm{MPa}$ caused by $\operatorname{pyr}\langle c+a\rangle$ and bas $\langle a\rangle$ slips. At the same time, the $\{00.2\} / /$ orientations continue to accumulate compressive strains, and these later develop large compressive strains and finally yield at $-320 \mathrm{MPa}$, corresponding to the beginning of $\operatorname{pyr}\langle c+a\rangle$ slip. At the end of the compressive test, these planes have different residual compressive strains, owing to the different plastic activity occurring in these different orientations, and the present EPSC model captures this trend correctly.

Surprisingly, the $\{00.2\} / /$ strains are very well reproduced by the $\operatorname{pyr}\langle c+a\rangle$ slip. As mentioned previously, the sharp reduction in the magnitude of the residual lattice strain in these grains is linked to twin activity $[8,39]$. Nevertheless, $\operatorname{pyr}\langle c+a\rangle$ is a competing mode in accommodating the strain along the $\langle c\rangle$ axis. Thus, the simulations indicate that the simultaneous operation of both ttw and $\operatorname{pyr}\langle c+a\rangle$ deformations modes is possible, with probably a predominant activity of tensile twin. In a recent work, Warwick et al. [40] used neutron diffraction to evaluate the evolution of lattice strains during compressive and tensile loadings of commercially pure titanium. The EPSC model used in this study accounts for the activation and relaxation due to twinning [39]. The sharp reduction in the magnitude of the compressive residual strain in the basal orientations for the compressed sample is reproduced by their model, and it is explained by the twin activity and the associated strain relaxation.

It can therefore be concluded that, while the current model deals well with the complexities of multiple slip modes, improvement in the twinning model is necessary for further work.

In the TD, the model accurately predicts the evolution of lattice strains in the majority of the 12 orientations studied. Nevertheless, some discrepancies are observed. For the $\{00.2\}_{\perp}$ orientation, the model captures the tensile state, although the final state is overestimated by $\sim 500 \mu \varepsilon$, probably due to a lack of stress relaxation description in the grains concerning by twin activity. For the $\{10.2\}_{\perp}$ and $\{11.2\}_{\perp}$ grains, the predictions are fairly accurate until $\sim-3 \%$, but fail to reproduce in magnitude the mechanical behaviour, even if they reproduce well the compressive strain in these orientations.

The residual lattice strains are also over-predicted in $\{10.3\}_{\perp}$ : very small experimental strains are recorded in this orientation, which is not predicted by the model after $-3 \%$ total strain. Perpendicularly to the tensile axis, the discrepancies between model calculations and measurements are more pronounced than in the $\{h k . l\}_{/ /}$grains.
This observation has already been made for the EPSC model [41]. The $\{h k . l\} / /$ orientations involve only similarly oriented grains in respect of the LD and are thus not so sensitive to slight variations in the representation of the crystallographic texture contrary to $\{h k . l\}_{\perp}$ grains. Despite these discrepancies, the model appears to reproduce correctly the main features of the lattice strain responses in the TD.

Fig. 7 shows plots of residual lattice strain vs. the orientation parameter $H^{2}$ in the longitudinal and transversal directions at $-3.16 \%$. $H^{2}$ represents the cosine of the angle between the prismatic $\{10.0\}$ plane and the basal $\{00.2\}$ plane.

A definite trend exists in the residual strain as a function of $H^{2}$, the details differ between the two directions. Along the longitudinal direction, the residual strains decrease monotonically from the $\{10.0\}$ pole $(407 \mu \varepsilon)$ to the $\{00.2\}$ pole $(-1146 \mu \varepsilon)$ with tensile strain for the lowest value of $H^{2}$ and compressive strains for $H^{2}>0.2$. In contrast, in the TD, the opposite behaviour is logically observed with compressive strains for $H^{2}<0.8$ and tensile values for higher values of $H^{2}$. At this strain level, the simulation gives very good agreement with the experimental results in trend and magnitude and also captures the change in lattice strains from tension to compression, whatever the direction. At this value of macroscopic deformation, the proportions of activated systems are: $50 \%$ for prismatic mode; $11 \%$ for pyramidal $\langle c+a\rangle ; 24 \%$ for pyramidal $\langle a\rangle$; $10 \%$ for basal slip; and $5 \%$ for tensile twinning.

(a)
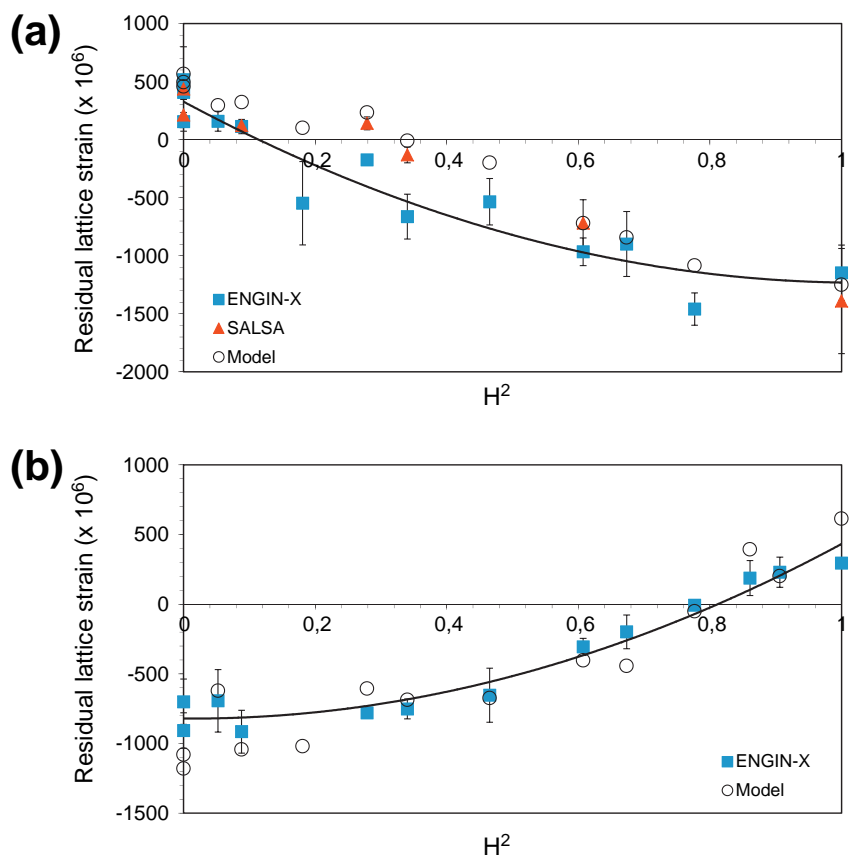

Fig. 7. Residual lattice strain vs. the orientation parameter $H^{2}$ from experimental data in the (a) longitudinal and (b) transversal directions. The predicted residual lattice strains values obtained by the models at $-3.16 \%$ total strain are also plotted. The lines serve as a guide for the eye. 


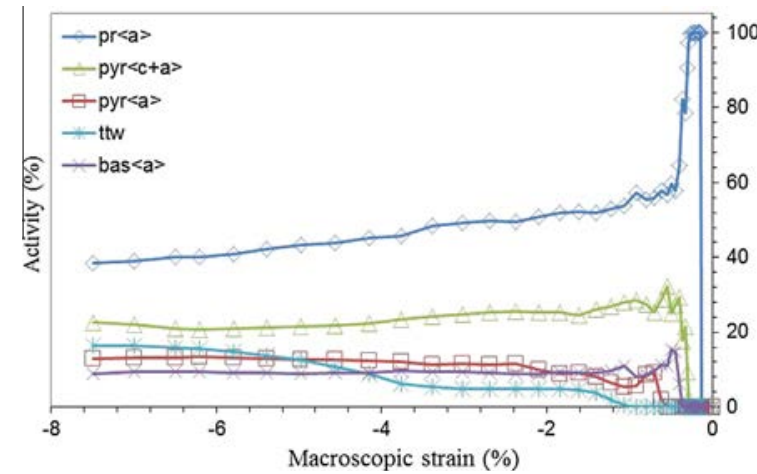

Fig. 8. Relative contribution of each deformation mode to the total shear as a function of deformation.

Plotting the corresponding evolution of the relative activities of the deformation modes (Fig. 8), it is clear that the crystallographic slip is the dominant mechanism of plastic deformation. Prismatic slip is always the primary mode, while pyramidal $\langle c+a\rangle$ slip is the next (39 and $22 \%$, respectively, at $-7.75 \%$ total strain). The relative contribution of twinning is $<20 \%$. After the compressive loads, the predicted twin volume fraction is $\sim 0.14$, close to that observed $(\sim 0.16)$.

Numerical results obtained at the different scales of the material show the relevance of this approach for hcp polycrystals.

\section{Conclusions}

The mechanical behaviour of a commercially pure titanium alloy was studied during uniaxial loading, taking into account the initial texture, the elastic and plastic anisotropies, slip and twin processes, work-hardening behaviour and texture evolution.

The present study highlights the usefulness of polycrystal models to explore the active deformation mechanisms in hexagonal alloys and the role that these mechanisms play in the global behaviour. To understand how internal strains evolve for textured material, a consistent data set (15 different reflections measured along the straining and TD for seven macroscopic prestrains) concerning the behaviour of a group of grains from a representative volume was obtained. These neutron experiments provide a comprehensive data set for testing the scale transition model developed for this study.

The choice of $\langle a\rangle$ slip as the main mechanism to accommodate plastic deformation can correctly explain the experimental strain values for the plane families studied. The model provides excellent results in predicting the macroscopic stress-strain curve. These predicted results were obtained for one single set of slip modes and hardening parameters, although it is not possible to consistently reproduce all the details of the residual lattice strain evolution, especially in the TD. The observed lattice strain relaxation in the $\{00.2\} / /$ orientations highlights the need for improvements to the twinning model in the future. The EPSC models are reasonably successful at reproducing the observed behaviour, but do not provide a complete description of the micromechanics of these materials, especially stress relaxation in twinning parent grains and the newly formed twin grains at the onset of twinning.

\section{Acknowledgements}

The authors thank the ISIS and ILL Neutron Facilities scientific committees for the allocated experimental days on the SALSA (experiment 1-02-30, ILL, France), Engin-X and GEM (experiment RB1010055, ISIS, UK) instruments. In particular, J. Kelleher, W. Kockelmann and T. Pirling are gratefully thanked for fruitful discussions and support.

\section{References}

[1] Partridge PG. Int Mater Rev 1967;12:169.

[2] Singh AK, Schwarzer RA. Z Metallkd 2000;91:702.

[3] Linga Murty K, Charit I. Prog Nucl Energy 2006;48:325.

[4] Gloaguen D, François M, Guillén R, Royer J. Acta Mater 2002;50:871.

[5] Pang JWL, Holden TM, Turner PA, Mason TE. Acta Mater 1999;47:373.

[6] Zattarin P, Lipinski P, Rosochowski A. Int J Mech Sci 2004;46:377.

[7] Baczmański A, Hfaiedh N, François M, Wierzbanowki K. Mater Sci Eng A 2009;501:153.

[8] Xu F, Holt RA, Daymond MR. Acta Mater 2008;56:3672

[9] Stapleton AM, Raghunathan SL, Bantounas I, Stone HJ, Lindley TC, Dye D. Acta Mater 2008;56:6186.

[10] Akhtar A. Met Trans 1975;6A:2201.

[11] Zaefferer S. Mater Sci Eng A 2003;344:20.

[12] Glavicic MG, Salemn AA, Semiatin SL. Acta Mater 2004;52:647.

[13] Tan XL, Gu HC. Mater Sci Eng 1994;189:77.

[14] Pochettino AA, Gannio N, Vial Edwards C, Penelle R. Scripta Metall 1992; $12: 1859$

[15] Salem A, Kalidindi SR, Doherty RD. Acta Mater 2003;51:4225.

[16] Akhtar A. Met Trans 1975;6A:1105.

[17] Chichili DR, Ramesh KT, Hemker KJ. Acta Mater 1998;46:1025.

[18] Pirling T, Bruno G, Withers PJ. Mater Sci Eng A 2006;437:139.

[19] Richard D, Ferrand M, Kearley GJ. J Neutron Res 1996;4:33.

[20] Santisteban JR, Daymond MR, James JA, Edwards L. J Appl Crystallogr 2006;39:812.

[21] Moreton-Smith CM, Johnston S, Akeroyd FA. J Neutron Res 1996;4:41.

[22] Kockelmann W, Chapon LC, Radelli PG. Physica B 2006;385:639.

[23] Hannon AC. Nucl Instrum Methods Phys Res 2005;551:88.

[24] Tenckhoff E. Deformation mechanisms, texture and anisotropy in zirconium and zircaloy. Philadelphia (PA): ASTM; 1988

[25] Brown DW, Beyerlein IJ, Sisneros TA, Clausen B, Tomé CN. Int J Plast 2012;29:120.

[26] Muransky O, Carr DG, Sittner P, Oliver EC. Int J Plast 2009;25:1107.

[27] Xu F, Holt MR, Daymond R, Rogge B, Oliver EC. Acta Mater 2008;488:172

[28] Kröner E. Acta Metall 1961;9:155.

[29] Hill R. J Mech Phys Solids 1965;13:89.

[30] Gloaguen D, Berchi T, Girard E, Guillén R. Acta Mater 2007:55:4369.

[31] Lipinski P, Berveiller M. Int J Plast 1989;5:149.

[32] Hutchinson W. Proc Roy Soc Lond A 1970;319:247.

[33] Zattarin P, Baczmanski A, Lipinski P, Wierzbanowski K. Arch Metall 2004;45:163. 
[34] Franz G, Abed-Meraim F, Lorrain JP, Ben Zineb T, Lemoine X, Berveiller M. Int J Plast 2009;25:205.

[35] Lorrain JP, Ben Zineb T, Abed-Meraim F, Berveiller M. Int J Forming Proc 2005;8:135.

[36] Gloaguen D, Berchi T, Girard E, Guillén R. Phys Status Solidi A 2006;203:R12.

[37] Simmons G, Wang H. Single crystal elastic constants and calculated aggregate properties. Cambridge (MA): MIT Press; 1971.
[38] Dye D, Stone HJ, Reed RC. Curr Opin Solid State Mater Sci 2001;5:31.

[39] Clausen B, Tomé CN, Brown DW, Agnew SR. Acta Mater 2008;56:2456.

[40] Warwick JLW, Jones NG, Rahman KM, Dye D. Acta Mater 2012;60:6720.

[41] Clausen B, Lorentzen T, Bourke MAM, Daymond MR. Mater Sci Eng A 1999;259:17. 